\title{
ACTIVIDAD DEL ACEITE ESENCIAL DE ALBAHACA (Ocimum basilicum) CONTRA Colletotrichum gloeosporioides DE ÑME (Dioscorea alata)
}

\section{ACTIVITY OF ESSENTIAL OIL OF BASIL (Ocimum basilicum) AGAINST Colletotrichum gloeosporioides OF YAM (Discorea alata)}

\author{
Alexander Pérez Cordero ${ }^{1}$, Deimer Vitola Romero ${ }^{2}$, Leonardo Chamorro Anaya ${ }^{3}$
}

${ }^{1} \mathrm{PhD}$. Universidad de Sucre, Facultad de Ciencias Agropecuarias, Grupo Bioprospección Agropecuaria. Campus Universitario Puerta Roja, A.A. 406, Sincelejo - Sucre, Colombia, e-mail: alexander.perez@unisucre.edu.co; ${ }^{2}$ Biólogo, Estudiante de Maestría en Ciencias Ambientales Sue-Caribe. Universidad de Sucre, Facultad de Educación y Ciencias, Grupo Bioprospección Agropecuaria. Campus Universitario Puerta Roja, A.A. 406, Sincelejo - Sucre, Colombia, e-mail: fitoquimicapn@gmail. com; ${ }^{3}$ M.Sc. Universidad de Sucre, Facultad de Educación y Ciencias, Grupo Bioprospección Agropecuaria. Campus Universitario Puerta Roja, A.A. 406, Sincelejo, Sucre, Colombia. e-mail: mscbiologia@gmail.com

\author{
Rev. U.D.CA Act. \& Div. Cient. 21(1): 99-108, Enero-Junio, 2018 \\ https://doi.org/10.31910/rudca.v21.n1.2018.667
}

\section{RESUMEN}

Los aceites esenciales de plantas aromáticas se han convertido, en los últimos años, en una práctica biológica, para controlar enfermedades causadas por organismos patógenos, en cultivos de interés agrícola, por lo tanto, en esta investigación, se evaluó in vitro la actividad inhibitoria de aceites esenciales, extraídos de hojas frescas de albahaca (Ocimum basilicum L.), contra Colletotrichum gloeosporioides (Penz.) Penz. y Sacc., agente causante de la antracnosis, en cultivos de ñame, en el departamento de Sucre, Colombia. Las hojas de albahaca fueron recolectadas en los municipios de Sincelejo, La Unión y Sampués, en septiembre y octubre de 2014; para la extracción de los aceites esenciales, se utilizaron hojas frescas, empleando el método de hidrodestilación asistida por microondas. De cada muestra, se prepararon concentraciones de 1, 1.000, 3.000, 5.000 y $10.000 \mathrm{ppm}$, de aceite esencial. Se utilizó un testigo absoluto, un control positivo con benomil $2,5 \mathrm{~g} / \mathrm{L}$ y un control negativo. Se realizó un diseño en bloque con arreglo factorial. En la actividad inhibitoria, se empleó la prueba de siembra directa en superficie y expresada como porcentaje de índice antifúngico. Los mayores porcentajes de índice antifúngico de los aceites esenciales obtenidos en las tres muestras, se observaron a 10.000 ppm, con $100 \%$ de inhibición. La actividad inhibitoria de los aceites esenciales de las tres muestras no mostró diferencias estadísticas, al compararlos con el control positivo. La caracterización química de los aceites esenciales, se realizó por cromatografía de gases, acoplado a espectrometría de masa, mostrando, como constituyente principal, al eugenol.
Palabras clave: Control biológico, hongo, alimento, planta medicinal.

\section{SUMMARY}

Essential oils of aromatic herbs have in recent years become a great biological tool for controlling diseases caused by pathogenic organisms. Therefore, in this research the inhibitory activity of essential oils of fresh leaves of Ocimum basilicum L. against Colletotrichum gloeosporioides (Penz.) Penz was evaluated in vitro agent causing the anthracnose in yam crops of the department of Sucre, Colombia. The leaves of $O$. basilicum were collected in the municipalities of Sincelejo, La Unión and Sampués in the months of september and october 2014; for the extraction of the essential oils, fresh leaves were used using the microwave assisted hydrodistillation method, concentrations of 1ppm, 1000ppm, 3000ppm, 5000ppm and 10000ppm of each essential oil were prepared, an absolute control, a control positive with benomil $2,5 \mathrm{~g} / \mathrm{L}$ and a negative control. A block design with factorial arrangement was employed. In the inhibitory activity the direct surface sowing test was used and was expressed as percentage of antifungal index. The highest percentages of \% I.A of these essential oils collected in the three municipalities were observed at a concentration of $10000 \mathrm{ppm}$, with an inhibition value of $100 \%$. The inhibitory activity of essential oils did not show significant differences when compared to the benomyl positive control. The chemical characterization of essential oils was performed by 
gas chromatography coupled to mass spectrometry, showing eugenol as the major constituent in each of the oils analyzed.

Key words: Biological control, fungus, food, medicinal plants.

\section{INTRODUCCIÓN}

Colombia es un país rico en recursos naturales, debido a las características de su relieve, clima, vegetación y clases de suelos. Esta condición favorece la siembra de diversos cultivos, entre los que se destaca el ñame (Dioscorea alata), cuya producción principal se concentra en la región Caribe, donde tiene gran importancia socioeconómica y cultural, puesto que sus tubérculos se usan, tanto para el consumo como para el abastecimiento del mercado local (Reina, 2012); no obstante, este cultivo es afectado por diversos agentes fitopatógenos, entre los que se destaca Colletotrichum gloeosporioides, causante de la antracnosis (Méndez et al. 2013). Esta enfermedad, se caracteriza por presentar lesiones necróticas en tallos, en hojas y en frutos, con amplia distribución en las regiones tropicales y subtropicales del mundo (Pinzón et al. 2013). C. gloeosporioides afecta numerosos cultivos y registra en Colombia un alto índice de daños agroeconómicos, en todas las áreas productoras. Los informes estadísticos indican pérdidas hasta de $100 \%$, según lo mencionado por Campo (2011), fuera de las condiciones de susceptibilidad de los clones, lo cual, conlleva un incremento en los costos de producción (Mendoza et al. 2007). Además, causa daños en cultivos de frutales de importancia económica y alimenticia, como los frutos de aguacate, banano, mango y tomate de árbol (Gañán et al. 2015), cereales, pastos, legumbres y cultivos perennes (Afanador et al. 2003).

En Colombia, esta enfermedad causó las mayores pérdidas registradas para el cultivo en el país, con una reducción del área sembrada, que pasó de 25.000ha, en 1989, a 4.547ha, en 1990, con una tasa de crecimiento promedio anual durante este periodo de, aproximadamente, $0,3 \%$, extremadamente baja, en comparación con 4,8\%, para 1990 (Reina, 2012).

El control de la antracnosis comprende el uso de semillas sanas, rotación de cultivos, poda y quema de material afectado, aspersiones con fungicidas, principalmente, benomil, manzeb, mancozeb, clorotalonil, captafol y folpet, biocontrol con hongos endófitos del género Nigrospora (Delgado \& Vásquez, 2010) y uso de especies resistentes, como $D$. rotundata y $D$. cayenensis, que presentaron resistencia a la enfermedad en campo (Méndez et al. 2013); la elección de las estrategias a implementar depende, en gran medida, de su disponibilidad (Aduramigba et al. 2008).
La alternativa más accesible y de rápida acción es el manejo químico; infortunadamente, la severidad de esta enfermedad ha conllevado a su uso excesivo de los fungicidas, antes mencionados, logrando, en principio, una disminución significativa de inóculo del patógeno, pero no obteniendo, en la mayoría de los casos, un control absoluto, por el contrario, induciendo problemas de resistencia en el patógeno (Aduramigba et al. 2008).

Los aceites esenciales, en los últimos años se han posicionado como una alternativa agronómica para sustituir, en parte, a los plaguicidas sintéticos del mercado actual, por poseer propiedades antifúngicas, antibacteriales, antivirales, entre otras. Además, pueden reemplazar a los aditivos sintéticos en los alimentos, favoreciendo la estabilidad de los mismos y protección contra las alteraciones lipídicas, por su actividad antioxidante. Estos son productos del metabolismo secundario de las plantas, cuya función es servir como barrera de defensa contra herbívoros, insectos y hongos (Bakkalli et al. 2008).

Se ha comprobado in vitro, la actividad del aceite esencial de O. basilicum contra Candida albicans, Alternaria sp., Penicillium digitatum, Escherichia coli, Pseudomonas auruginosas, mientras que el extracto acuoso es activo contra Staphylococcus aureus (Cáseres, 1993).

Esta investigación tuvo como objetivo evaluar in vitro, la actividad inhibitoria de aceites esenciales, de tres ecotipos de albahaca contra C. gloeosporioides, causante de la antracnosis, en ñame.

\section{MATERIALES Y MÉTODOS}

Área de toma de muestras: El área de muestreo estuvo comprendida en tres municipios del departamento de Sucre, Colombia: 1) Municipio de La Unión, perteneciente a la subregión San Jorge, ubicado a una latitud Norte de 851'20,2” y longitud Oeste de 75¹6'27, 1" a 63 msnm. Presenta zonas de bosque húmedo, seco y muy seco tropical, así como sabanas naturales, precipitación promedio anual de $2.300 \mathrm{~mm}$, temperatura promedio de $28^{\circ} \mathrm{C}$ y humedad relativa del $85 \%$ (Aguilera, 2005); 2) Municipio de Sincelejo, que hace parte de la subregión Montes de María, con una latitud Norte de 9॰17'12,92" y longitud Oeste de $75^{\circ} 22^{\prime} 43,2^{\prime \prime}$, a 187 m.s.n.m. Presenta bosque seco tropical, donde predomina la niebla y con montañas de 700 m.s.n.m., por lo que son productores de agua; la temperatura promedio está entre 20 a $30^{\circ} \mathrm{C}$, precipitación anual promedio de $1.500 \mathrm{~mm}$ y humedad relativa entre 75 y 85\% (Aguilera, 2013) y 3) Municipio de Sampués, subregión Sabana, a latitud Norte de $9^{\circ} 11^{\prime} 31,1^{\prime \prime}$ y longitud Oeste de $75^{\circ} 22^{\prime} 45,3^{\prime \prime}$, a 145 m.s.n.m. Se inicia a partir del declive de los Montes de María hasta inicios de la depresión 
del bajo Cauca y San Jorge, conformado por numerosas sierras y colinas, formando ondulaciones, que van desde los 70 hasta $185 \mathrm{~m}$.s.n.m.; es la subregión que posee con mayor intensidad la estación seca y su clima es característico de zonas de bosque seco tropical, con pocos relictos de vegetación secundaria y predominio de paisaje de lomerío; por la fuerte intervención humana, se le conoce como sabanas antrópicas, cuya temperatura promedio anual es de $27,2^{\circ} \mathrm{C}$, la precipitación promedio anual fluctúa entre 990 y $1.275 \mathrm{~mm}$ y la humedad relativa es de $80 \%$ (Aguilera, 2005).

Recolección del material vegetal: Las muestras fueron colectadas durante octubre de 2014. Los tejidos vegetales utilizados para la extracción de aceites fueron hojas frescas de plantas silvestre de albahaca; las hojas, se tomaron de la parte media y superior de los especímenes. Las plantas, se encontraban en etapa de floración y en buen estado fitosanitario; se colectaron durante la puesta del sol, para evitar que las temperaturas altas pudieran volatizar algunos componentes presentes en las hojas. El material vegetal, se empacó en un contenedor de polietileno expandido y se preservó a $25^{\circ} \mathrm{C}$, de acuerdo con la metodología propuesta por Granados et al. (2012). La identificación taxonómica de las plantas fue realizada en el herbario de la Universidad de Sucre, registro nacional de colecciones biológicas.

Procesamiento del material: Las hojas fueron lavadas con agua y seleccionadas, para garantizar su buen estado; seguidamente, se trozaron, se pesaron y se sometieron al proceso de extracción, de acuerdo con la metodología propuesta por Vitola \& Pérez (2016).

Extracción de aceites esenciales: La extracción de los aceites esenciales, se realizó por el método de hidrodestilación asistida por microondas (MWHD), propuesto por Vitola \& Pérez (2016). Se empleó un equipo de hidrodestilación, con capacidad para $2 \mathrm{~L}$, en el cual, se introdujeron $300 \mathrm{~g}$ de hojas, con $250 \mathrm{~mL}$ de agua destilada, en el balón de extracción y calentado por $30 \mathrm{~min}$, divididos en tres ciclos de $10 \mathrm{~min}$; como fuente de radiación microondas, se empleó un horno convencional. Los aceites esenciales, se colectaron en un aparato tipo Dean Stark; el aceite esencial, se separó por decantación e, inmediatamente, fue deshidratado con sulfato de sodio anhidro y almacenado a $4^{\circ} \mathrm{C}$ en frascos de vidrio, tipo ámbar, hasta su posterior utilización.

Rendimiento de la extracción de aceites esenciales: Para determinar el rendimiento, se utilizó la fórmula: $R=(-$ $\mathrm{V} / \mathrm{M}) * 100$, en donde: $\mathrm{R}=$ rendimiento $(\%), \mathrm{V}=$ volumen del aceite esencial $(\mathrm{mL})$ y $M=$ masa del material vegetal $(\mathrm{g})$ (Pino et al. 2012).

Hongo: Correspondió a la cepa identificada como Colletotrichum gloeosporioides (Penz.) Penz. \& Sacc, la cual, fue identificada y donada por la Universidad de los Andes, Bogotá; esta, a su vez, se encuentra en el grupo de investigación en Bioprospección Agropecuaria de la Universidad de Sucre, para posteriores investigaciones.

Evaluación in vitro de la actividad antifúngica del aceite: La prueba de actividad inhibitoria contra C. gloeosporioides, se realizó siguiendo lo propuesto por Vitola \& Pérez (2016), utilizando el método de siembra directa, donde un segmento de $6 \mathrm{~mm}$ del hongo fue sembrado en cajas Petri, conteniendo papa-dextrosa-agar (PDA) (extracto de patata 4g/L, Dextrosa $20 \mathrm{~g} / \mathrm{L}$, Agar-Agar 15g/L ajustado a pH: 5,5 $\pm 0,2$ ), enriquecido con antibióticos (Cloranfenicol 20mg/L, Ampicilina 50mg/L y Rifampicina $10 \mathrm{mg} / \mathrm{L})$. Se emplearon cinco concentraciones del aceite esencial: 1, 1,000, 3,000, 5,000 y 10,000ppm. A cada caja, se les adicionó $35 \mu \mathrm{L}$ de las concentraciones, disuelta en solución de Tween 20 al 0,5\% y dimetilsulfoxido (DMSO), al 0,2\% en agua. Se utilizó un control positivo con benomil y un testigo absoluto sin ningún tipo de tratamiento. Se realizó un diseño en bloque completamente al azar con arreglo factorial. Las cajas, se incubaron a $30 \pm 2^{\circ} \mathrm{C}$, por 8 días, en intervalos de $12 \mathrm{~h}$ de luz y $12 \mathrm{~h}$ de oscuridad. La actividad antifúngica, se evaluó midiendo el crecimiento radial del hongo con las diferentes concentraciones después del día octavo. El resultado, se interpretó como porcentaje de índice antifúngico: Ïndice antifúngico $(\%)=\left[1-\left(\mathrm{D}_{a} / \mathrm{D}_{\mathrm{b}}\right)\right]$ x 100 , en donde: $\mathrm{D}_{\mathrm{a}}$, corresponde al crecimiento del hongo en cada tratamiento y $\mathrm{D}_{\mathrm{b}}$, al crecimiento del testigo absoluto (Guo et al. 2008). Para determinar la eficiencia de cada aceite, se restó el porcentaje de Índice antifúngico del control negativo al porcentaje de Índice antifúngico de cada aceite esencial y se comparó con el control positivo (benomil).

Análisis de aceites esenciales por cromatografía de gases acoplado a espectrometría de masa (CG/EM): La determinación de los componentes de los aceites esenciales, se efectuó por cromatografía de gases, acoplada a espectrometría de masa (GC/EM), utilizando un cromatógrafo de gases Agilent $6890 \mathrm{~N}$, acoplado a un detector selectivo de masa Agilent 5973N. Los índices de Kóvats fueron determinados en una columna capilar DB_5MS $(30 \mathrm{mx} 320 \mu \mathrm{mx} 0,5 \mu \mathrm{m})$, utilizando, como gas de arrastre, el Helio, con una presión de $0.27 \mathrm{psi}$ y un promedio de velocidad de flujo de $40 \mathrm{~cm} / \mathrm{s}$. La temperatura inicial del horno fue de $150^{\circ} \mathrm{C}$ y la final, $350^{\circ} \mathrm{C}$. La temperatura del inyector fue de $250^{\circ} \mathrm{C}$ y la del detector, $300^{\circ} \mathrm{C}$. La identidad de los componentes, se asignó por comparación del espectro de masas, obtenido experimentalmente para cada componente, con los reportados en las bases de datos de NIST98.L, NIST02.L y NIST5a.L.

Análisis estadístico: Se realizó un diseño de experimento en bloques completamente al azar, con arreglo factorial $5 \times 3$, con tres réplicas por tratamiento, usando el programa estadístico Statgraphics Centurión XVI.I versión 16.1.18 (StatPoint Tech- 
nologies, Inc., 2010). Se realizó la prueba de Tukey, para comparar la diferencia entre la media de los niveles de los factores.

\section{RESULTADOS Y DISCUSIÓN}

Clasificación científica de los ejemplares vegetales: Las muestras recolectadas en los municipios de Sincelejo, La Unión y Sampués correspondieron a albahaca (O. basilicum), especie perteneciente a la familia Lamiaceae, la cual, se encuentra en la colección botánica del herbario de la Universidad de Sucre, bajo registro $\mathrm{N}^{\circ} 000829$.

Rendimiento de la extracción de aceites: El rendimiento del aceite esencial extraído de hojas frescas de albahaca colectadas en el municipio de Sincelejo fue de $0,21 \%$, el de Sampués, 0,20\% y de La Unión, 0,19\%.

De acuerdo con Fonnegra (2007), el rendimiento de aceite esencial de albahaca en Colombia, se encuentra en un intervalo de 0,04 hasta 0,7\%. Estudios realizados por Beltrán et al. (2013) indican que de las especies estudiadas del género Ocimum, el mayor rendimiento que se obtuvo fue de la especie cinammom, con un $0,324 \%$, seguido por album y thyrsiflorum, con valores de 0,158 y $0,145 \%$, respectivamente. Es de indicar que el rendimiento de los aceites esenciales depende de muchos factores, como la localización geo-climática, el tipo de suelo, el estado de desarrollo de la planta (antes, durante o después de la floración) e, inclusive, la hora del día cuando se cosecha, entre otros. Los factores geo-climáticos y el tipo de suelo pueden dar origen a diferentes quimiotipos de la planta, de los cuales, se destilan aceites esenciales con composición química, propiedades sensoriales y actividad biológica diferentes (Hussain et al. 2008).

Actividad antifúngica de los aceites: Se hallaron diferencias estadísticas significativas ( $p$-valor $<0,05)$ entre los aceites esenciales evaluados, así como entre las concentracio- nes, mostrando el aceite esencial de hojas de O. basilicum, colectado en el municipio de Sincelejo, los mayores promedios de actividad inhibitoria contra el crecimiento micelial de C. gloeosporioides (Tablas 1 y 2). Además, la concentración de 10,000ppm fue la que presentó el mayor porcentaje de índice antifúngico, con una inhibición de 100\% (Gráfico 1), efecto similar al observado con el control químico con benomil (Figura 1); no obstante, los aceites esenciales obtenidos de hojas de O. basilicum, colectada en los municipios de La Unión y Sampués, no mostraron diferencias estadísticas significativas ( $p$-valor $>0,05)$ con respecto a los índices de inhibición contra dicho fitopatógeno. Estos resultados demuestran que los aceites esenciales obtenidos de esta especie vegetal, procedente de los tres municipios, tuvieron actividad antifúngica contra C. gloeosporioides; sin embargo, es necesario realizar estudios in vivo, que permita demostrar efecto antifúngico, similar al reportado, en condiciones de laboratorio, así como evaluar los posibles efectos sobre la biota benéfica del suelo, su fitotoxicidad, la foto y biodegradabilidad de estos aceites esenciales, para poder considerarlos plenamente como amigables con el medio ambiente y, aún más importante, como agente de control de este fitopatógeno en campo.

Varios autores han encontrado que extractos vegetales y aceites esenciales de plantas tienen potencial antifúngico contra Colletotrichum sp; por ejemplo, Pérez et al. (2011) reportaron actividad inhibitoria de aceites extraídos del árbol del paraíso (Melia azederach) sobre este hongo. Alzate et al. (2009) evaluaron la actividad inhibitoria de aceites esenciales de tomillo (Thymus vulgaris) y limoncillo (Cymbopogon citratus) contra $C$. acutatum del árbol de tomate y encontraron inhibición del $100 \%$ en la germinación de las esporas; asimismo, Pérez et al. (2017) evaluaron la actividad inhibitoria del aceite esencial de limoncillo contra C. gloesporioides y encontraron actividad inhibitoria del $100 \%$, contra este hongo.

Tabla 1. Análisis de varianza para porcentaje de índice antifúngico.

\begin{tabular}{|c|c|c|c|c|c|}
\hline Fuente & $\begin{array}{l}\text { Suma de } \\
\text { Cuadrados }\end{array}$ & GL & Cuadrado Medio & Razón-F & Valor-P \\
\hline \multicolumn{6}{|l|}{ EFECTOS PRINCIPALES } \\
\hline A: Localidad & 715,16 & 2 & 357,58 & 122,93 & 0.0000 \\
\hline B: Concentración & 52659,9 & 4 & 13165,0 & 4525,78 & 0.0000 \\
\hline \multicolumn{6}{|l|}{ INTERACCIONES } \\
\hline $\mathrm{AB}$ & 424.655 & 8 & 53.0819 & 18,25 & 0.0000 \\
\hline RESIDUOS & 87.2667 & 30 & 2,90889 & & \\
\hline TOTAL (CORREGIDO) & 53887,0 & 44 & & & \\
\hline
\end{tabular}




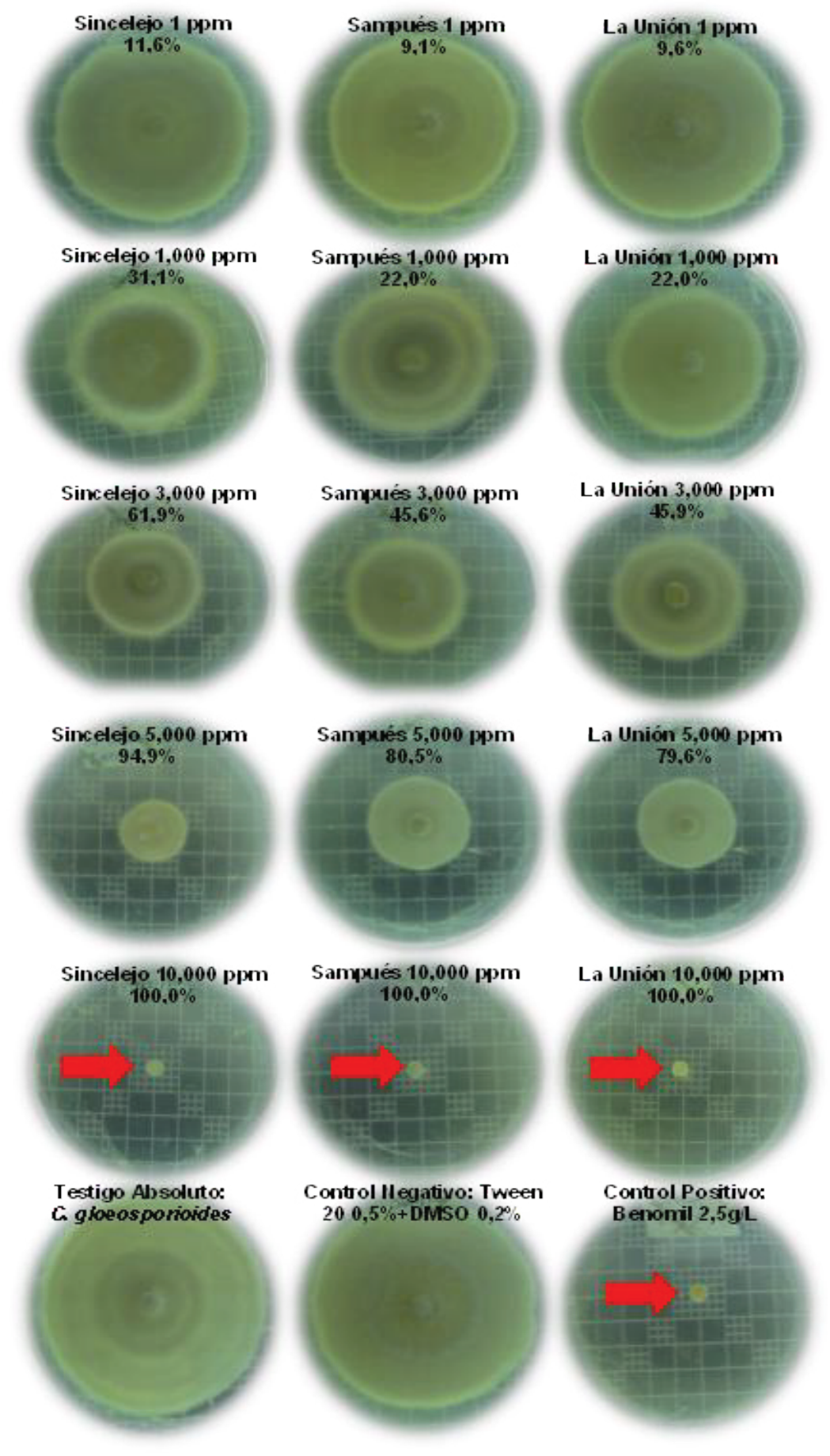

Figura 1. Actividad inhibitoria (\%) in vitro, de cinco concentraciones de aceites esenciales de O. basilicum contra C. gloeosporioides, de plantas colectadas en los municipios de Sincelejo, Sampués y La Unión, del departamento de Sucre. 
Tabla 2. Prueba múltiple de rango (HSD) Tukey para la variable respuesta porcentaje de índice antifúngico por zona de colecta y concentración.

\begin{tabular}{|l|l|l|l|l|}
\hline \multicolumn{1}{|c|}{ Zona de Colecta } & \multicolumn{1}{|c|}{ Casos } & \multicolumn{1}{c|}{ Media LS } & \multicolumn{1}{c|}{ Sigma LS } & \multicolumn{1}{c|}{ Grupos Homogéneos } \\
\hline La Unión & 15 & 51,42 & 0,44037 & A \\
\hline Sampués & 15 & 51,4533 & 0,44037 & A \\
\hline Sincelejo & 15 & 59,8933 & 0,44037 & B \\
\hline Concentración (ppm) & Casos & \multicolumn{1}{c|}{ Media LS } & \multicolumn{1}{c|}{ Sigma LS } & Grupos Homogéneos \\
\hline 1 & 9 & 10,1 & 0,568516 & A \\
\hline 1.000 & 9 & 25,0333 & 0,568516 & B \\
\hline 3.000 & 9 & 51,1444 & 0,568516 & C \\
\hline 5.000 & 9 & 85,0 & 0,568516 & D \\
\hline 10.000 & 9 & 100,0 & 0,568516 & E \\
\hline
\end{tabular}

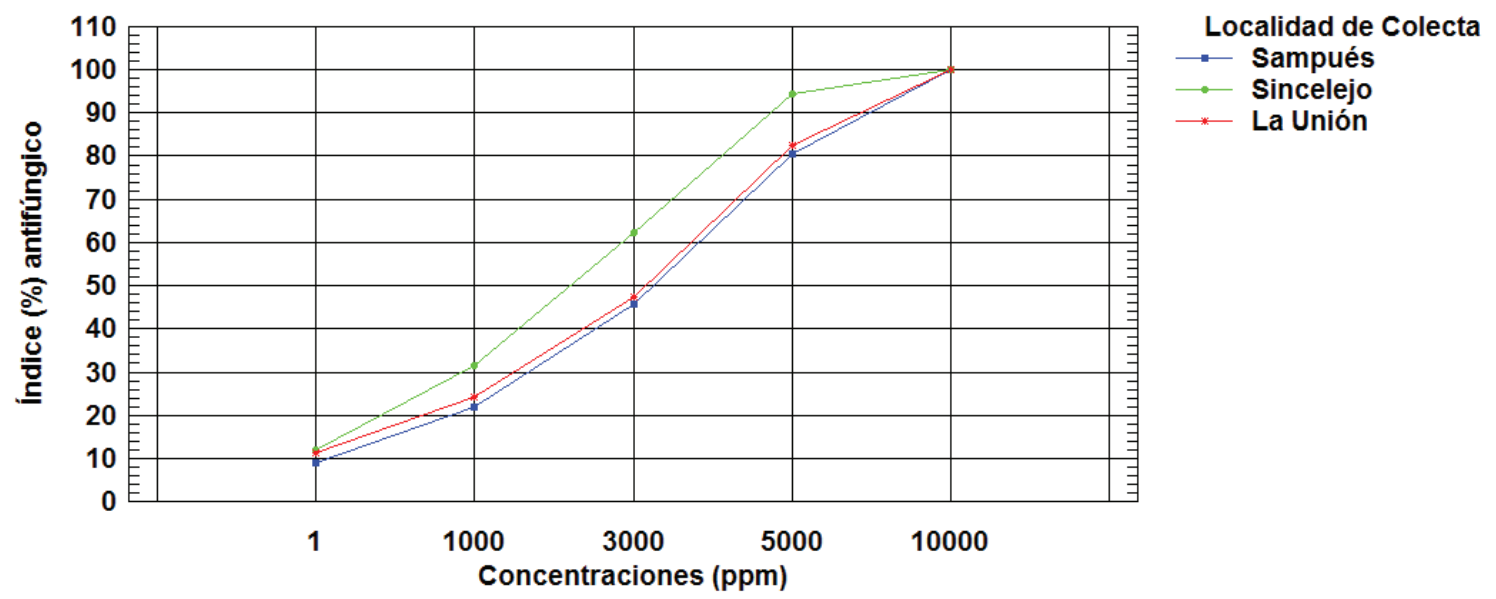

Gráfico 1. Interacción entre los factores evaluados.

Análisis químico: Cromatografía de gases acoplado a espectrometría de masa: La albahaca (O. basilicum) es una especie vegetal medicinal aromática que tiene un alto contenido de aceites esenciales, rico en eugenol, de amplio uso en la medicina (Sam et al. 2002). Se ha reportado actividad antifúngica contra hongos, como: Aspergillus niger, Mucor mucedo, y Fusarium solani, entre otros (Hussain et al. 2008). Además, en estudios realizados por Hanif et al. (2010), se ha confirmado la presencia de otros aceites esenciales, como linalool $(69,9 \%)$, geraniol $(10,9 \%)$ y 1,8 -cineole $(6,4 \%)$, entre otros.

En este estudio, se pudo corroborar la presencia de eugenol como metabolito secundario de los aceites esenciales de las muestras obtenidas, recolectadas en los tres municipios, con concentraciones de $75,44,45,15$ y $80,28 \%$, respectivamente (Tabla 3 ). A este principio activo, le han reportado propiedades farmacológicas importantes, como la de presentar actividad antimicrobiana, antiagregante y antipirética (Laekeman et al. 1990); asimismo, actividad antianafiláctica, porque puede inhibir la degranulación de las células cebadas que provocan hipersensibilidad (Kim et al. 1997), lo cual, concuerda con los resultados de este estudio, al mostrar actividad antimicrobiana contra C. gloeosporioides, a 10,000ppm. A pesar, que el aceite esencial de las muestras procedentes de Sampués fue el que presentó mayor concentración de este alilbenceno, con $80,28 \%$, tuvo el menor porcentaje de actividad inhibitoria $(9,1 \%)$ a la concentración de $1 \mathrm{ppm}$, lo que demuestra que la concentración antifúngica óptima de este metabolito secundario, contenido en el aceite esencial de esta especie vegetal contra C. gloeosporioides, es de alrededor del $75 \%$; aunque, los tres aceites esenciales tuvieron actividad fungicida a 10,000ppm. Los resultados del análisis por cromatografía muestran como metabolito 
Tabla 3. Perfiles químicos por CG/EM de los aceites esenciales de O. basilicum, procedentes de tres municipios del departamento de Sucre.

\begin{tabular}{|c|c|c|c|c|c|c|c|c|c|}
\hline \multirow{2}{*}{$\mathrm{Pk} \#$} & \multicolumn{3}{|c|}{ Sincelejo } & \multicolumn{3}{|c|}{ Sampués } & \multicolumn{3}{|c|}{ La Unión } \\
\hline & TR & Área (\%) & Identificación & TR & Área (\%) & Identificación & TR & Área (\%) & Identificación \\
\hline 1 & 5,007 & 0,03 & Pineno & 3,339 & 0,04 & 3-Hexen-1-ol & 3,559 & 0,08 & 3-Hexen-1-ol \\
\hline 2 & 5,310 & 0,03 & Camfeno & 5,006 & 0,05 & $\alpha$-Pineno & 5,007 & 0,14 & Pineno \\
\hline 3 & 7,165 & 0,07 & Eucaliptol & 5,310 & 0,05 & Camfeno & 5,310 & 0,13 & $\begin{array}{l}\text { Camfeno+Artemisia } \\
\text { trieno }\end{array}$ \\
\hline 4 & 9,046 & 0,10 & No & 5,898 & 0,06 & $\beta$-Pireno & 5,905 & 0,11 & $\alpha, \beta$-Pireno \\
\hline 5 & 9,214 & 0,05 & No & 6,616 & 0,15 & No & 6,286 & 0,03 & No \\
\hline 6 & 10,693 & 0,36 & Borneol & 7,165 & 0,28 & Eucaliptol & 6,609 & 0,13 & No \\
\hline 7 & 17,233 & 75,44 & Eugenol & 8,192 & 0,04 & $\begin{array}{l}\text { 1-Octanol + } \\
\text { Ácido Fórmico, } \\
\text { octil ester }\end{array}$ & 7,165 & 0,19 & Eucaliptol \\
\hline 8 & 18,015 & 16,28 & Cariofileno & 8,735 & 0,05 & No & 7,714 & 0,21 & No \\
\hline 9 & 18,325 & 0,23 & No & 9,052 & 0,16 & Linalol & 8,192 & 0,06 & No \\
\hline 10 & 18,629 & 1,17 & $\alpha$-Cariofileno & 10,713 & 0,45 & Borneol & 8,735 & 0,03 & No \\
\hline 11 & 18,926 & 0,28 & No & 11,501 & 0,04 & No & 9,045 & 0,19 & Linalool \\
\hline 12 & 19,243 & 0,37 & Eremofileno & 14,403 & 0,07 & Tridecano & 9,213 & 0,03 & No \\
\hline 13 & 19,424 & 0,26 & No & 17,149 & 80,28 & Eugenol & 10,267 & 0,06 & Alcanfor \\
\hline 14 & 19,799 & 2,13 & $\beta$-Elemene & 17,976 & 12,44 & Cariofileno & 10,719 & 0,89 & Borneol \\
\hline 15 & 19,967 & 0,07 & No & 18,312 & 0,14 & No & 16,955 & 45,15 & Eugenol \\
\hline 16 & 20,141 & 0,16 & No & 18,616 & 0,92 & $\alpha$-Cariofileno & 17,433 & 23,11 & $\begin{array}{l}(+) \text {-Aromadendreno } \\
+\beta \text {-Elemene }\end{array}$ \\
\hline 17 & 20,296 & 0,21 & No & 19,243 & 0,29 & $\alpha$-Guaieno & 18,118 & 20,15 & Cariofileno \\
\hline 18 & 20,768 & 0,87 & $\alpha$-Elemol & 19,424 & 0,24 & No & 18,338 & 0,19 & No \\
\hline 19 & 21,783 & 0,76 & No & 19,773 & 1,55 & $\beta$-Elemene & 18,635 & 1,39 & $\alpha$-Cariofileno \\
\hline 20 & 22,894 & 0,13 & No & 20,128 & 0,13 & No & 18,894 & 0,22 & No \\
\hline 21 & 23,017 & 0,05 & $\beta$-Cubebeno & 20,277 & 0,05 & No & 19,236 & 0,52 & Eremofileno \\
\hline 22 & 23,450 & 0,28 & No & 20,755 & 0,65 & $\begin{array}{l}\text { Elemol + Hedi- } \\
\text { cariol }\end{array}$ & 19,430 & 0,53 & No \\
\hline 23 & & & & 19,844 & 2,15 & $\begin{array}{l}\beta \text {-Elemene }+ \\
\text { Eremofileno }\end{array}$ & & & \\
\hline 24 & & & & 19,980 & 0,04 & No & & & \\
\hline 25 & & & & 20,122 & 0,08 & No & & & \\
\hline 26 & & & & 20,264 & 0,05 & No & & & \\
\hline 27 & & & & 20,755 & 0,71 & $\alpha$-Elemol & & & \\
\hline 28 & & & & 21,401 & 0,06 & No & & & \\
\hline 29 & & & & 23,934 & 0,27 & Óxido Cariofileno & & & \\
\hline 30 & & & & 25,808 & 0,03 & No & & & \\
\hline 31 & & & & 30,164 & 0,04 & $\begin{array}{l}\text { 9,17Octadecadi- } \\
\text { enal, (Z)- }\end{array}$ & & & \\
\hline
\end{tabular}

Pk \#: picos arrojados en el cromatograma; TR: Tiempo de Retención de cada metabolito; No: no identificado con la base de datos utilizada. 
secundario mayoritario el Eugenol, con $61,78 \%$, para los aceites esenciales de $O$. basilicum, colectados en los tres municipios, objetos de estudio. Similares resultados encontraron Özcan \& Chalchat (2002) y Dev et al. (2011), mientras que Ismail (2006), Hussain et al. (2008) y Hanif et al. (2010) hallaron, como metabolito mayoritario, en esta misma especie vegetal, el linnalol.

El aceite esencial de albahaca procedente de Sincelejo y de La Unión presentaron otros compuestos, como el eremofileno, mientras que el aceite esencial procedente de muestras de Sampués presentó al isómero guaieno. Además, el aceite esencial de las plantas recolectadas en Sincelejo contiene los compuestos hidicariol, $\beta$-cubebeno y $\gamma$-cadineno, mientras que el aceite esencial de las muestras recolectadas en La Unión no presentó ninguno de estos metabolitos y el de Sampués, solo contenía hidicariol. Es de resaltar que solo el aceite esencial obtenido de las plantas de La Unión contenía aromadendreno.

Las diferencias químicas encontradas en los aceites esenciales de esta especie vegetal influenciaron, de una u otra manera, el efecto contra el hongo en estudio, resultado que fue muy satisfactorio, por su potencial dentro de un manejo integrado de C. gloeosporioides, en el cultivo del ñame. Estas diferencias químicas y su potencial inhibitorio, posiblemente, se deben a las diferentes condiciones de los ecosistemas, de donde fueron recolectadas las muestras. Los factores climáticos, como el régimen de precipitaciones, puede alterar el rendimiento en la producción de eugenol, presente en el aceite esencial de albahaca. Al comparar la concentración de eugenol encontrada en los aceites esenciales en cada uno de los tres ecotipos y el régimen de precipitación en las subregiones de colecta, se encontró que son variables inversamente proporcionales, puesto que en las muestras procedentes del municipio de La Unión la concentración de eugenol fue de $45,15 \%$, con una precipitación promedio anual de $2.300 \mathrm{~mm}$; en las de Sincelejo, la concentración de este aceite fue de $75,44 \%$, con una precipitación promedio anual de $1.500 \mathrm{~mm}$ y en las del municipio de Sampués, la concentración de eugenol fue de $80,28 \%$, con una precipitación promedio anual de $990 \mathrm{~mm}$. Esto indica, posiblemente, que la producción de eugenol es más eficiente cuando los regímenes de precipitaciones son bajos y, por consiguiente, la planta es sometida a estrés hídrico; caso contrario ocurre cuando la planta se halla en ecosistema, en donde el volumen de precipitación es más alto, produciendo menor cantidad de eugenol.

Los aceites esenciales evaluados mostraron una bioactividad importante contra C. gloesporioides, con potencial para ser evaluados in vivo. Cabe resaltar que durante los 8 días de la prueba antagónica solo se realizó una aplicación de los tratamientos, por lo cual, se recomienda, a posteriores investiga- ciones, realizar dos o tres aplicaciones durante este mismo periodo, esperando un efecto antagónico, en corto tiempo, contra este fitopatógeno. Por lo tanto, la importancia de los problemas fitosanitarios demanda un cambio urgente de manejo agrícola, por lo que es necesario desarrollar nuevas alternativas, que coadyuven al desarrollo agrosostenible del ambiente. Esta problemática es posible solucionarla, a través de un manejo integrado de las enfermedades; es allí, donde la agroecología juega un rol importante, por propiciar el equilibrio entre los humanos y la naturaleza, adoptando un sistema de producción, que permita mejorar la calidad de los suelos y obtener productos limpios, para el consumo humano.

Agradecimientos: A los integrantes del grupo de investigación Bioprospección Agropecuaria de la Universidad de Sucre. Conflicto de intereses: Este manuscrito es original y fue preparado, revisado con la participación de todos los autores, quienes declaramos que no existe conflicto de intereses que ponga en riesgo la validez de los resultados del mismo. Financiación: Este trabajo fue financiado por el grupo de investigación Bioprospección Agropecuaria de la Universidad de Sucre.

\section{BIBLIOGRAFÍA}

1. ADURAMIGBA, O.; ASIEDU, R.; ODEBODE, C. 2008. Reaction of Dioscorea alata (water yam) to anthracnose disease in Nigeria. J. Food Agric. Environ. 6:248-252.

2. AFANADOR, L.; MINZ, D.; MAYMOND, M.; FREEMAN, S. 2003. Characterization of Colletotrichum isolates from Tamarillo, Passiflora, y Mango in Colombia and identification of a unique species of the genus. Phytopathology. (USA) 93(5):579-587.

3. AGUILERA, M. 2005. La economía del departamento de Sucre: ganadería y sector público. Banco de República de Colombia y centros de estudios económicos regionales (CEER). Cartagena. p.18-23.

4. AGUILERA, M. 2013. Montes de María: una subregión de economía campesina y empresarial. Banco de República de Colombia y centros de estudios económicos regionales (CEER). Cartagena. p.20-21.

5. ALZATE, D.; MIER, G.; AFANADOR, L.; DURANGO, D.; GARCÍA, C. 2009. Evaluación de la fitotoxicidad y la actividad antifúngica contra Colletotrichum acutatum de los aceites esenciales de tomillo (Thymus vulgaris), limoncillo (Cymbopogon citratus), y sus componentes mayoritarios. Vitae. (Colombia). 16(1):116-125. 
6. BAKKALLI, F.; AVERBECK, S.; AVERBECK, D.; IDAOMAR, M. 2008 Biological Effects of Essential Oils-a Review. Food Chem. Toxicol. (Inglaterra). 46(2):446475.

7. BELTRÁN, C.; CANTILLO, P.; VIVAS, M. 2013. Actividad antibacteriana de los aceites obtenidos de Ocimum basilicum L. var. cinammom, O. album, O. thyrsiflorum, para uso potencial en fitocosmética. Investig. Andina. (Colombia). 15(27):798-810.

8. CAMPO, O. 2011. Manejo integrado de la antracnosis (Colletotrichum spp.) en ñame (Dioscorea alata), mediante el uso de alternativas para reducir el inoculo primario, la dispersión y el establecimiento del patógeno. Informe final 2008-2011. Facultad de Ciencias Agrícolas de la Universidad de Córdoba, Colombia.

9. CÁSERES, A. 1993. Plantas de uso medicinal en Guatemala. Editorial Universidad San Carlos (Ciudad de Guatemala). 402p.

10. DELGADO, E.; VÁSQUEZ, S. 2010. Control biológico de la Antracnosis causada por Colletotrichum gloeosporioides (Penz. y Sacc.) en Tomate de Árbol (Solanum betaceum Cav.) mediante hongos endófitos antagonistas. La Granja. (Ecuador). 11(1):36-43.

11. DEV, N.; DAS, K.; HOSSAIN, A.; RAHMAN, M. 2011. Chemical compositions of different extracts of Ocimum basillicum leaves. J. Sci. Res. (Bangladesh). 3(1):197-206.

12. FONNEGRA, R. 2007. Plantas medicinales aprobadas en Colombia. 2. ed. Medellín: Editorial Universidad de Antioquia. (Colombia). 368p.

13. GAÑÁN, L.; ÁLVAREZ, E.; CASTAÑO-ZAPATA, J. 2015. Identificación genética de aislamientos de Colletotrichum spp. causantes de antracnosis en frutos de aguacate, banano, mango y tomate de árbol. Rev. Acad. Colomb. Cienc. Ex. Fis. Nat. 39(152):339347.

14. GRANADOS, C.; YÁÑEZ, X.; SANTAFÉ, G. 2012. Evaluación de la actividad antioxidante del aceite esencial foliar de Calycolpus moritzianus y Minthostachys mollis de Norte de Santander. Bistua. (Colombia). 10(1):12-23.

15. GUO, Z.; XING, R.; LIU, S.; ZHONG, Z.; JI, X.; WANG, L.; LI, P. 2008. The influence of molecular weight of quaternized chitosan on antifungal activity. Carbohydr. Polym. (Reino Unido). 71(4):694-697.

16. HANIF, A.M.; AL-MASKARI, Y.M.; AL-MASKARI, A.; AL-SHUKAILI, A.; AL-MASKARI, Y.A.; AL-SABAHI, N.J. 2010. Essential oil composition, antimicrobial and antioxidant activities of unexplored Omani basil. J. Med. Plants Res. (Nigeria). 5(5):751-757.

17. HUSSAIN, A.; ANWAR, F.; HUSSAIN, S.; PRZYBYLSKI, R. 2008. Chemical composition, antioxidant and antimicrobial activities of basil (Ocimum basilicum) essential oils depends on seasonal variations. Food Chem. (Reino Unido). 108(3):986-995.

18. ISMAIL, M. 2006. Central properties and chemical composition of Ocimum basilicum essential oil. Pharm. Biol. (USA). 44(8):619-626.

19. KIM, M.; LEE, H.; KIM, Y.; CHUNG, G.; KIM, H.; LIM, P.; SHIN, Y. 1997. Antianaphylactic properties of eugenol. Pharmacol. Res. (Italia). 36(6):475-80.

20. LAEKEMAN, M.; VAN HOOF, H.; VANDEN BERGHE, A.; HERMAN, G.; VLIETINCK, J. 1990. Eugenol a valuable compound for in vitro experimental research and worthwhile for further in vivo investigation. Phytother. Res. (USA). 4(3):90-96.

21. MÉNDEZ, Y.; PALENCIA, J.; HERNÁNDEZ, K.; HERNÁNDEZ, E.; BELTRÁN, J. 2013. Reacción de genotipos de ñame (Dioscorea spp) a la antracnosis (Colletotrichum gloeosporioides). Tema Agrarios. (Colombia). 18(1):34-40.

22. MENDOZA, C.; MORENO, C.; WEIL, M.; ELANGO, F. 2007. Evaluación del efecto de extractos vegetales sobre el crecimiento in vitro de Phytophthora palmivora Bult y Colletotrichum gloeosporioides (Penz) Penz. \& Sacc. Tierra Trop. (USA). 3(1):81-89.

23. ÖZCAN, M.; CHALCHAT, C. 2002. Essential oil composition of Ocimum basilicum L. and Ocimum minimum L. in Turkey. Czech J. of food Sc. (República Checa). 20(6):223-228.

24. PÉREZ, A.; CHAMORRO, L.; VITOLA, D.; HERNÁNDEZ, J. 2017. Actividad antifúngica de Cymbopogon citratus contra Colletotrichum gloeosporioides. Agron. Mesoam. (Costa Rica). 28(2):465-475.

25. PÉREZ, A.; ROJAS, J.; CHAMORRO, L.; PÉREZ, K. 2011. Evaluación in vitro de actividad inhibitoria de extractos vegetales sobre cepas de hongos del géne- 
ro Colletotrichum sp. Acta Agronómica (Colombia). 60(2):158-164.

26. PINO, O.; SÁNCHEZ, Y.; ROJAS, M.M.; ABREU, Y.; CORREA, T.M. 2012. Composición química y actividad antibacteriana del aceite esencial de Pimpinella anisum L. Rev. Protección Veg. (Cuba). 27:181-187.

27. PINZÓN, Y.; BUSTAMANTE, S.; BUITRAGO, G. 2013. Diagnóstico molecular diferencial de Colletotrichum gloeosporioides y Fusarium oxysporum en ñame (Dioscorea sp.). Rev. Col. Biotecn. (Colombia). 15(1):52-60.

28. REINA, Y. 2012. El cultivo del ñame en el caribe colombiano. Centro de Estudios Económicos Regionales (CEER)-Cartagena (Ed.). Documento de trabajo sobre economía regional. 168(2):1-34.
29. SAM, O.; DE LA LUZ, M.; BARROSO, L. 2002. Caracterización anatómica de las hojas de la albahaca blanca (Ocimum basilicum L.). Rev. Cultivos Tropicales. (Cuba). 23(2):39-42.

30. STATPOINT TECHNOLOGIES, Inc. 2010. Statgraphics ${ }^{\circledR}$ Centurion XVI.I versión 16.1.18. disponible URL: www.STATGRAPHICS.com.

31. VITOLA. D.; PÉREZ, A. 2016. Bioactividad de extracto de Annona muricata y aceites esenciales de Citrus aurantium contra Phytophthora cinnamomi. Recia. (Colombia). 8(Supl):325-334.

Recibido: Agosto 22 de 2017

Aceptado: Mayo 24 de 2018

Cómo citar:

Pérez Cordero, A.; Vitola Romero, D.; Chamorro Anaya, L. 2018. Actividad del aceite esencial de albahaca (Ocimum basilicum) contra Colletotrichum gloeosporioides de ñame (Dioscorea alata). Rev. U.D.C.A Act. \& Div. Cient. 21(1): 99-108. 